\title{
Dementia Care in the Time of COVID-19 Pandemic
}

\author{
Babak Tousi ${ }^{\mathrm{a}, \mathrm{b}, 1, *}$ \\ ${ }^{a}$ Head, Clinical Trials Program, Lou Ruvo Center for Brain Health, Neurological Institute, \\ Cleveland Clinic, Cleveland, $\mathrm{OH}, \mathrm{USA}$ \\ ${ }^{\mathrm{b}}$ Associate Professor of Medicine and Neurology, Cleveland Clinic Lerner College of Medicine- \\ Case Western Reserve University, Cleveland, OH, USA
}

Accepted 19 June 2020

\begin{abstract}
Patients with dementia are particularly vulnerable during the COVID-19 pandemic. The initial response to COVID19 promoted behavioral changes in both society and healthcare, while a long-term solution is sought by prioritizing societal values. In addition, there has been disruption to clinical care and clinical research. This pandemic might have significantly changed the care for our patients with dementia toward increased acceptance of telemedicine by the patients and providers, and its utilization in both clinical care and research.
\end{abstract}

Keywords: Access, Alzheimer's disease, caregivers, clinical trial, coronavirus, COVID-19, dementia, health care, pandemic, telehealth, telemedicine

In recent months, the COVID-19 pandemic has changed the world dramatically. The pandemic shined a spotlight on the weaknesses of not only the health care systems but also the financial fragility of society. The initial response to COVID-19 promoted behavioral changes while a long-term solution is sought by prioritizing societal values. COVID19 required a quick shift from traditional models of health care delivery to innovative problem solving.

While the pandemic has become a challenge for all families, it is additionally burdensome for families who are dealing with a chronic neurodegenerative condition that affects cognition. Increased age, commonly seen among dementia patients, is considered

\footnotetext{
${ }^{1}$ Present address: Cleveland Clinic Lou Ruvo Center for Brain Health, Cleveland, OH, USA.

*Correspondence to: Babak Tousi MD, FACP, Cleveland Clinic, Lou Ruvo center for Brain Health, 9500 Euclid Avenue, U-10, Cleveland, OH 44195, USA. Tel.: +1 216237 6400; Fax: +1 216237 6415; E-mail: Batous@ccf.org.
}

a predictor of poor outcome of hospitalization for COVID-19 patients [1]. A British study of 16,749 hospitalized patients with COVID-19 found a diagnosis of dementia also adds a risk factor unfavorable to the outcome with $39 \%$ higher risk of death [2].

Early in the pandemics some physicians in Italy came up with a strict criterion to allocate ventilators and ICU beds for patients who are less than 80 years of age and not having comorbidities such as advanced dementia [3]. The criterion was implemented due to shortage of ventilators and giving priority to the ones who are most likely to benefit.

The act has been followed in the United States. Some state's emergency protocol initially labeled advanced dementia and severe traumatic brain injury as poor candidates for ventilator support. Age has an important effect on outcome for mechanical ventilation $[4,5]$, but categorical exclusions based on one criterion were not supported in the medical literature [6]. These guidelines have sparked criticism and 
backlash which led to alternative guidelines such as the University of Pittsburgh Medical Center (UPMC) policy which is based on a point scale assessing the odds of recovery. Sequential Organ Failure Assessment (SOFA) and other points are allocated based on the patients having conditions with life expectancy $<1$ year or $<5$ years. The initial guideline (March 2020) specified Alzheimer's or related dementias as condition with shorter life expectancy but diagnosis by itself was not exclusionary [7]. The authors removed the list of example conditions in the revised version (April 2020) to avoid the risk of the list being used as a blanket judgment instead of considering an individual's overall clinical condition [8]. Pennsylvania and few other institutions adopted this scoring system to allocate critical care during a public health emergency $[8,9]$.

COVID-19 disease, which was initially thought to primarily affect the respiratory tract and lungs, has now emerged with an array of neurological symptoms in more than one third of the patients. Symptoms span cognitive impairment, dizziness, ataxia to seizures, meningoencephalitis, and stroke [10-12]. In some cases, neurological symptoms were the initial presentation without dyspnea in spite of lung involvement [13]. It remains to be seen if these manifestations are due to direct effect of virus infiltrating the nervous system or secondary to inflammatory response of the body. The sequence of severe respiratory distress with potential hypoxia to the central nervous system may have a late effect on cognition and executive function as well. Alzheimer's Association emphasized that increased confusion for a patient with dementia is often the first symptom of any illness in their recent COVID-19 tips for caregivers [14].

Over the past decades, few respiratory viruses were demonstrated to have neuro-invasive capabilities with potential long-term sequelae. Parkinsonism was a late feature of encephalitis lethargica following the influenza pandemic of 1918 [15]. Coronaviruses can be neuro-invasive in humans and animals and were suggested to persist in the central nervous system of humans and of mice that survive acute encephalitis $[16,17]$. The presence of Coronaviruses in the brain may have pathological consequences in some vulnerable individuals causing long-term sequelae by triggering or attributing to neurodegenerative and neuro-inflammatory diseases [16]. It is not known if COVID-19 can establish a latent infection in the nervous system as some coronaviruses may do. A recent autopsy case series of ten patients with COVID-19 did not reveal any central nervous system involvement and polymerase chain reaction testing was negative in all cerebrospinal fluid samples, but the authors did not specify if there was any clinical neurological symptoms prior to death [18]. Large scale registry studies will provide information on long term neurological outcomes of COVID-19.

\section{CHANGE IN CAREGIVING}

Over the past few months, society has settled into new norms of social distancing. Social distancing is a phrase we are all familiar with now, but it is misleading, as it is physical distancing rather than social distance that aids in prevention of transmission of the virus. Society is used to gathering for many social interactions, but we do not need to be physically gathered to be socially connected. As societal norms change, "distancing" safeguards are necessary to contain the virus but may cause discomfort and turbulence in daily living that exacerbates mental health issues.

Faced with uncertainty, there are exercises we can do to protect from overwhelming depression and anxiety: get facts from credible sources, break down information logically, don't dwell on worst case scenarios and best scenarios, writing down your thoughts, maintain connection on social media, or talking to someone you trust. These strategies will improve caregiver resilience and mental health especially when incorporated with regular sleep, regular exercise, hydration, balanced nutrition, limiting alcohol intake, and limiting media exposure.

Patients with dementia may not understand changes in their routine and become agitated because their regular routine is important, providing predictability and comfort. The ability to discuss COVID-19 with a dementia patient depends on the stage of cognitive impairment. If the patient is capable of comprehending the building blocks of the information and how to help them protect themselves and not become upset, then a conversation about the pandemic is realistic. If the patient would be frightened or delusional about a potentially fatal disease, the conversation will be futile, possibly even harmful. Caregivers have to judge how information will impact the person's behavior.

Patients with advanced dementia have limited comprehension, so social distancing may cause anxiety which means it falls on the caregivers to assume the responsibility to keep them safe using protocols recommended by the Centers for Disease Control 
and Prevention (CDC) and tips provided by the Alzheimer's Association [14].

People can have strong emotions manifest during a shelter-in period. Individuals who might normally be with a caregiver may not understand why they are alone and isolated. It is important to communicate with the patient and help them recognize strong emotions and how to deal with them. The strength we feel by providing kindness and support to others and receiving it in return is powerful. Caring for a loved one can be challenging, but during a time of emergency it is even more challenging. Self-care for caregivers is important too, as they take care of loved ones, so they too need to maintain a healthy perspective and balance.

As a resource, many communities have an aging agency that may provide resources and support. During any crisis, personal care is requested more, so evaluating available resources and having back up plans is important. A financial resource through the Federal government, the Coronavirus Aid, Relief and Economic Security (CARES) act provides additional funding for family caregiver support. The bill's Pandemic Unemployment Assistance provision will allow qualifying individuals to claim unemployment in the event they are unable to work or telework due to the need to provide ongoing home care to an individual in the household who is unable to attend facilities, including adult care programs, "that are closed as a direct result of the COVID-19 and such facility care is required for the individual to work" [19]. The bill also covers the individual who is unable to work due to providing care for a family member who has been diagnosed with COVID-19.

Uncertainty fuels anxiety, which leads to less rational decisions. During pandemics, opportunists are rife with scams. COVID-19 will likely see a spike in unsavory individuals attempting to take advantage of vulnerable people, especially elderly and those with dementia and other cognitive impairments. Caregivers should maintain daily contact and ask questions so that any phishing scams or changes in major financial activities can be identified and thwarted immediately.

\section{CHANGE IN PROVIDING CARE}

The first noticeable change to providing care for patients with dementia was to change from the traditional model of office visit to virtual visits. The telemedicine model of care has been used in the Unites States healthcare institutions before. The Cleveland Clinic Health System launched its telemedicine in 2014 and saw a rapid increase in the use of virtual visit over past couple years to 41,000 virtual visits in 2019 [20], but its widespread use has been limited by low reimbursement, interstate licensing and practice issues [21]. This number increased dramatically to about 200,000 visits per month since the pandemic.

The silver lining of the recent pandemic maybe globalization of telemedicine. Physician's visits have turned into virtual visits conducted over computers, tablets, or smartphones, a pattern which may continue beyond the stay-in period. Having a reliable device to communicate with healthcare providers for the patient and the family becomes a necessity.

Dementia management has been shown to be quite amenable to virtual evaluation [22]. Cognitive assessment is strongly dependent on interview and questioning rather than direct physical examination. One study showed that patients and caregivers reported similar satisfaction with virtual visits and office visits [23].

Therapy services also adapted to the practice of telemedicine. The appropriate referrals for a visit may include cognitive rehabilitation, exercise training, ADL assessment, speech/swallowing interventions, and even gait assessment and training. Certainly, these therapies will be limited by the state licensure laws that may require the patients to reside in the state where the service is provided.

The Centers for Medicare \& Medicaid (CMS) policy for telemedicine services reimbursement has changed for the duration of the COVID-19 Public Health Emergency [24]. Medicare will make payment for Medicare telemedicine services provided to patients in the patients' places of residence. The CMS temporarily waived requirements that practitioners be licensed in the state where they are providing services but this waiver does not have the effect of waiving state or local licensure requirements [25]. The efficiency of this method, especially in the elderly population, raises hope that the CMS policy for telemedicine services becomes a permanent CMS policy for Medicare patients in the United States and hopefully other insurers. The challenge lies in the barriers that seniors face when adopting to a new technology due to cognitive, physical, and financial challenges or lack of familiarity. Only four in ten seniors own a smartphone [26]. 80\% of 65- to 69year-old adults are Internet users, while less than half of seniors above age 80 use the Internet [26]. Other 
family members may be able to provide a tablet or smartphone for the time of visits but in some cases, it will be limited to a telephone visit. Telephone visits do not provide the capability of video examination or some cognitive assessments and are reimbursed at lower rate.

\section{DEMENTIA RESEARCH IN THE TIME OF PANDEMICS}

One impact from sudden change to "shelter-in" status is the operation of clinical trials and observational studies. Pharmaceutical sponsors have tried to uphold the integrity of the clinical trials, while finding new ways to adapt to necessary changes. Many trials allow missing visits or skipping trial medication to some degree. Some trials allow the medication to be shipped to the participant's house directly with remote monitoring, such as virtually counting the remaining pills, while others may try to arrange for a third party to provide injection or infusion at home. Trial sites have explored ways to conduct trial assessments and coordinate care remotely through the help of telemedicine. That also puts extra burden on research staff when addressing local regulatory committees, in line with regulatory organizations such as FDA guidelines.

If the halt due to COVID-19 is temporary, that may not affect the integrity of data collection. However, if the disruption is long-lived, the interpretation of the results due to missing data or quality of data collection can affect the integrity of the study. So far, the FDA and NIH are open to granting protocol waivers and extensions. The FDA has issued guidance on the conduct of clinical trials to assist sponsors in assuring the safety, compliance with good clinical practice, and trial integrity for the duration of the COVID-19 public health emergency [27].

The transformation of healthcare opens the door to new opportunities such as using digital biomarkers in measuring or monitoring the functions at home. The concept of measuring activities during a pandemic in the early detection of a change in health status in the elderly is not new [28]. Digital biomarkers are still in the early stage of validation to be used as the primary outcome. Design of future clinical trials may include digital biomarkers as outcome measures which should undergo the same formal regulatory process as traditional biomarkers for approval.

COVID-19 maybe a novel virus but throughout the centuries, our ancestors have survived different pandemics without our current technology. It may give us a sense of strength knowing that we are not the first to undergo tribulations.

\section{DISCLOSURE STATEMENT}

The author's disclosure is available online (https:// www.j-alz.com/manuscript-disclosures/20-0461r2).

\section{REFERENCES}

[1] Zhou F, Yu T, Du R, Fan G, Liu Y, Liu Z, Xiang J, Wang Y, Song B, Gu X, Guan L, Wei Y, Li H, Wu X, Xu J, Tu S, Zhang Y, Chen H, Cao B (2020) Clinical course and risk factors for mortality of adult inpatients with COVID-19 in Wuhan, China: A retrospective cohort study. Lancet 395, 1054-1062.

[2] Docherty AB, Harrison EM, Green CA, Hardwick HE, Pius R, Norman L, Holden KA, Read JM, Dondelinger F, Carson G, Merson L, Lee J, Plotkin D, Sigfrid L, Halpin S, Jackson C, Gamble C, Horby PW, Nguyen-VanTam JS, Dunning J, Openshaw PJM, Baillie JK, Semple MG (2020) Features of 16,749 hospitalised UK patients with COVID-19 using the ISARIC WHO Clinical Characterisation Protocol. medRxiv, 2020.04.23.20076042; doi: https://doi.org/10.1101/2020.04.23.20076042

[3] Cipriani G, Di Fiorino M (2020) Access to care for dementia patients suffering from COVID-19. Am J Geriatr Psychiatry, doi: 10.1016/j.jagp.2020.04.009

[4] Feng Y, Amoateng-Adjepong Y, Kaufman D, Gheorghe C, Manthous CA (2009) Age, duration of mechanical ventilation, and outcomes of patients who are critically ill. Chest 136, 759-764.

[5] Cohen IL, Lambrinos J (1995) Investigating the impact of age on outcome of mechanical ventilation using a population of 41,848 patients from a statewide database. Chest $\mathbf{1 0 7}$, 1673-1680.

[6] White DB, Lo B (2020) A framework for rationing ventilators and critical care beds during the COVID-19 pandemic. JAMA, doi: 10.1001/jama.2020.5046

[7] White, Douglas B (2020) Allocation of Scarce Critical Care Resources During a Public Health Emergency [Internet]. Available from: https://ccm.pitt.edu/sites/default/files/Mod el-hospital-policy-for-allocation-of-critical-care_2020-0323-web.pdf

[8] University of Pittsburgh (2020) Allocation of Scarce Critical Care Resources During a Public Health Emergency [Internet]. Available from: https://ccm.pitt.edu/?q= content/model-hospital-policy-allocating-scarce-critical-ca re-resources-available-online-now

[9] Pennsylvania Department of Health, The Hospital \& Healthsystem Association of Pennsylvania (2020) Interim Pennsylvania Crisis Standards of Care for Pandemic Guidelines [Internet]. Available from: https://www.health.pa.gov/ topics/Documents/Diseases-and-Conditions/COVID-19-In terim-Crisis-Standards-of-Care.pdf

[10] Mao L, Wang M, Chen S, He Q, Chang J, Hong C, Zhou Y, Wang D, Li Y, Jin H, Hu B (2020) Neurological manifestations of hospitalized patients with COVID-19 in Wuhan, China: A retrospective case series study. medRxiv, 2020.02.22.20026500; doi: https://doi.org/ $10.1101 / 2020.02 .22 .20026500$ 
[11] Oxley TJ, Mocco J, Majidi S, Kellner CP, Shoirah H, Singh IP, De Leacy RA, Shigematsu T, Ladner TR, Yaeger KA, Skliut M, Weinberger J, Dangayach NS, Bederson JB, Tuhrim S, Fifi JT (2020) Large-vessel stroke as a presenting feature of Covid-19 in the young. N Engl J Med 382, e60.

[12] Moriguchi T, Harii N, Goto J, Harada D, Sugawara H, Takamino J, Ueno M, Sakata H, Kondo K, Myose N, Nakao A, Takeda M, Haro H, Inoue O, Suzuki-Inoue K, Kubokawa K, Ogihara S, Sasaki T, Kinouchi H, Kojin H, Ito M, Onishi H, Shimizu T, Sasaki Y, Enomoto N, Ishihara H, Furuya S, Yamamoto T, Shimada S (2020) A first case of meningitis/encephalitis associated with SARS-Coronavirus-2. Int $J$ Infect Dis 94, 55-58.

[13] Zhao H, Shen D, Zhou H, Liu J, Chen S (2020) GuillainBarré syndrome associated with SARS-CoV-2 infection: Causality or coincidence? Lancet Neurol 19, 383-384.

[14] Alzheimer's Association (2020) Coronavirus (COVID-19): Tips for Dementia Caregivers.

[15] Cheyette SR, Cummings JL (1995) Encephalitis lethargica: Lessons for contemporary neuropsychiatry. J Neuropsychiatry Clin Neurosci 7, 125-134.

[16] Desforges M, Le Coupanec A, Dubeau P, Bourgouin A, Lajoie L, Dubé M, Talbot PJ (2020) Human coronaviruses and other respiratory viruses: Underestimated opportunistic pathogens of the central nervous system? Viruses 12, 14.

[17] Arbour N, Day R, Newcombe J, Talbot PJ (2000) Neuroinvasion by human respiratory coronaviruses. $J$ Virol $\mathbf{7 4 ,}$ 8913-8921.

[18] Schaller T, Hirschbühl K, Burkhardt K, Braun G, Trepel M, Märkl B, Claus R (2020) Postmortem examination of patients with COVID-19. JAMA 323, 2518-2520.

[19] United States Department of Labor (2020) Pandemic Unemployment Assistance (PUA) Implementation and Operating Instructions [Internet]. Available from: https://wdr.doleta. gov/directives/attach/UIPL/UIPL_16-20_Attachment_1.pdf

[20] Cleveland Clinic (2020) Celebrating 100,000 Outpatient Virtual Visits [Internet]. Available from: https://consultqd. clevelandclinic.org/celebrating-100000-outpatient-virtualvisits/
[21] Cleveland Clinic (2019) The Future of Telemedicine (and What's in the Way) [Internet]. Available from: https://consultqd.clevelandclinic.org/the-future-of-telemed icine-and-whats-in-the-way/

[22] Hatcher-Martin JM, Adams JL, Anderson ER, Bove R, Burrus TM, Chehrenama M, Dolan O'Brien M, Eliashiv DS, Erten-Lyons D, Giesser BS, Moo LR, Narayanaswami P, Rossi MA, Soni M, Tariq N, Tsao JW, Vargas BB, Vota SA, Wessels SR, Planalp H, Govindarajan R (2020) Telemedicine in neurology: Telemedicine Work Group of the American Academy of Neurology update. Neurology 94, 30-38.

[23] Morgan DG, Crossley M, Kirk A, McBain L, Stewart NJ, D'Arcy C, Forbes D, Harder S, Dal Bello-Haas V, Basran J (2011) Evaluation of telehealth for preclinic assessment and follow-up in an interprofessional rural and remote memory clinic. J Appl Gerontol 30, 304-331.

[24] Centers for Medicare \& Medicaid (2020) Medicare Telemedicine Health Care Provider Fact Sheet [Internet]. Available from: https://www.cms.gov/newsroom/fact-she ets/medicare-telemedicine-health-care-provider-fact-sheet

[25] The Centers for Medicare and Medicaid Services (CMS) (2020) COVID-19 Emergency Declaration Blanket Waivers for Health Care Providers. pp. 1-40.

[26] Anderson M, Perrin A (2017) Technology use among seniors. Pew Research Center. Available from: https://www. pewresearch.org/internet/2017/05/17/technology-use-amon g-seniors/

[27] U.S. Food and Drug Administration (2020) FDA Guidance on Conduct of Clinical Trials of Medical Products during COVID-19 Public Health Emergency [Internet]. Available from: https://www.fda.gov/regulatory-information/searchfda-guidance-documents/fda-guidance-conduct-clinicaltrials-medical-products-during-covid-19-public-healthemergency

[28] Campbell IH, Austin D, Hayes TL, Pavel M, Riley T, Mattek $\mathrm{N}$, Kaye J (2011) Measuring changes in activity patterns during a norovirus epidemic at a retirement community. Conf Proc IEEE Eng Med Biol Soc 2011, 6793-6796. 